\title{
NEW RIFAMYCINS PRODUCED BY A RECOMBINANT STRAIN OF NOCARDIA MEDITERRANEI
}

\author{
T. Schupp, P. Traxler and J. A. L. Auden \\ Pharmaceuticals Division of Ciba-Geigy Limited, \\ Basel, Switzerland
}

(Received for publication April 27, 1981)

\begin{abstract}
A recombinant strain of Nocardia mediterranei was found to produce a number of new rifamycins which are structurally related to rifamycin $\mathrm{S}$, rifamycin $\mathrm{W}$ and rifamycin $\mathrm{G}$. This strain was derived from two Nocardia mediterranei mutants by intraspecific recombination.
\end{abstract}

In the search for new derivatives of antibiotics one possible approach is to manipulate the producing strain genetically and analyse its fermentation products. There are three effective ways of creating new genotypes in actinomycetes: mutation, recombination and transfer of plasmids.

Previous studies have shown that mutation of Nocardia mediterranei can lead to new biosynthetic variants of the rifamycin molecule. By this means rifamycin $\mathrm{SV}^{1)}$, rifamycin $\mathrm{W}^{2)}$ and protorifamycin $I^{3)}$ were found, all biosynthetic precursors of rifamycin B, as well as a considerable number of other new rifamycins $s^{4,5,6,7)}$.

The occurrence of genetic recombination in Nocardia mediterranei has been demonstrated ${ }^{8)}$ and we used this process to screen for new rifamycins. In the course of this work strain R-21 was found, which was derived from intraspecific recombination between two Nocardia mediterranei mutants, one producing rifamycin $\mathrm{B}$ and the other rifamycin W. This strain ${ }^{9)}$ was found to produce a number of new rifamycins (Fig. 1) in addition to the known rifamycins $\mathrm{B}, \mathrm{O}, \mathrm{S}, \mathrm{W}, \mathrm{P}$. In this and the following paper ${ }^{10)}$ we describe the<smiles>COC(C(C)C(C)C=CC=CC(C)C)C(C)C(O)C(C)C(O)C(C)/C=C/C=C(/C)C(=O)Nc1cc(OC(=O)O)c2c(C(=O)O)c(OC(C)C)c(C)c(O)c2c1O</smiles>

Rifamycin B isolation and characterization of recombinant R-21 and the purification and structural elucidation of the new rifamycins it produces.

\section{Materials and Methods}

\section{Strains}

All strains of Nocardia mediterranei used for this work were either mutational or recombinant derivatives from a culture of Nocardia mediterranei ATCC 13685. The auxotrophic and streptomycin resistance markers used have been previously described ${ }^{8)}$. Mutant strain T191 is blocked in the transformation of rifamycin $\mathrm{W}$ to rifamycin $\mathrm{S}$ and accumulates rifamycin $\mathrm{W}$ in the fermentation broth.

\section{Media}

The minimal (MM) and complete agars $(\mathrm{CM})$ were as previously described ${ }^{8)}$. They were used for maintenance of the cultures and the crossing procedure. The liquid medium NL 148 ${ }^{3)}$ was used to screen the recombinant strains for production of new rifamycins. Another liquid medium, NL $151 \mathrm{~b}^{32}$, 
Fig. 1. New rifamycins isolated from strain R-21.

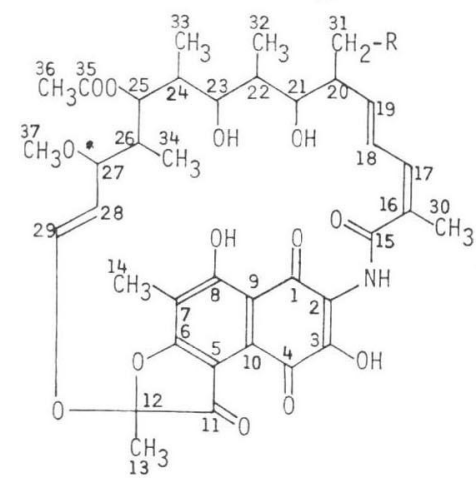

$\mathrm{R}=\mathrm{H}:$ 3-Hydroxyrifamycin $\mathrm{S}$

$\mathrm{R}=\mathrm{OH}:$ 3,31-Dihydroxyrifamycin $\mathrm{S}$

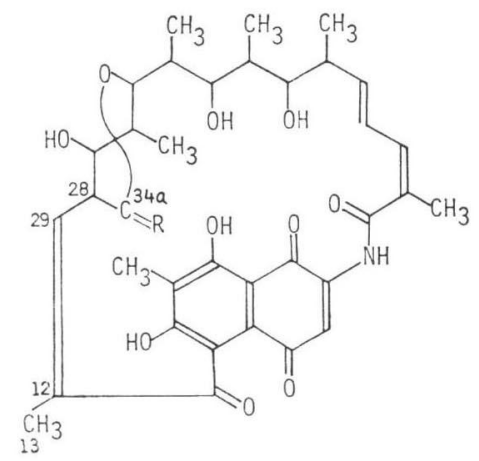

$\mathrm{R}=\mathrm{O}$ : Rifamycin W-lactone

$\mathrm{R}=\mathrm{H}, \mathrm{OH}:$ Rifamycin W-hemiacetal<smiles>COC(/C=C/C=C/[14C](C)C(C)C(C(C)=O)C(C)C(O)C(C)C(O)C(C)/C=C/[14C](=O)Nc1cc(=O)c2c3c(c(C)c(O)c2o1)OC(C)(O)O3)C(C)=O</smiles>

16,17-Dehydrorifamycin G

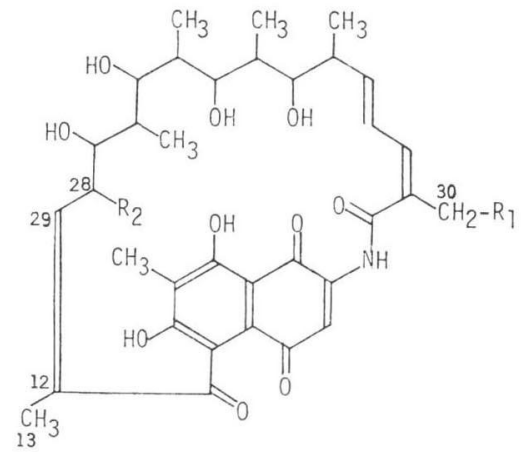

$\mathrm{R}_{1}=\mathrm{OH}, \mathrm{R}_{2}=\mathrm{CH}_{2} \mathrm{OH}$ : 30-Hydroxyrifamycin $\mathrm{W}$ $\mathrm{R}_{1}=\mathrm{OH}, \mathrm{R}_{2}=\mathrm{OH}$ : 28-Dehydroxymethyl-28,30dihydroxyrifamycin $\mathrm{W}$

$\mathrm{R}_{1}=\mathrm{H}, \mathrm{R}_{2}=\mathrm{CH}_{2} \mathrm{OH}$ : Rifamycin W

was used to obtain sufficient quantities of the new rifamycins produced by strain R-21. Medium 148c was used as preculture medium for the 300 liters fermentation (seed culture) and contained: Peanut meal $7.5 \mathrm{~g} /$ liter, glucose $5 \mathrm{~g} /$ liter, $\mathrm{KH}_{2} \mathrm{PO}_{4} 0.33 \mathrm{~g} /$ liter, $\mathrm{MgSO}_{4} \cdot 7 \mathrm{H}_{2} \mathrm{O} 0.33 \mathrm{~g} /$ liter, $\mathrm{FeSO}_{4} \cdot 7 \mathrm{H}_{2} \mathrm{O}$ $0.0033 \mathrm{~g} /$ liter, $\mathrm{ZnSO}_{4} \cdot 7 \mathrm{H}_{2} \mathrm{O} 0.0165 \mathrm{~g} /$ liter, $\mathrm{MnSO}_{4} \cdot 4 \mathrm{H}_{2} \mathrm{O} 0.0013 \mathrm{~g} /$ liter, $\mathrm{CaCO}_{3} 1.65 \mathrm{~g} /$ liter, antifoams (UCON LB $6250.125 \mathrm{~g} /$ liter, SAG $4710.375 \mathrm{~g} /$ liter).

\section{Crossing Procedure}

Parental cultures, each derived from a purified clone, were grown for 4 days in liquid medium 148 ( $20 \mathrm{ml}$ per Erlenmeyer flask). Eight $\mathrm{ml}$ of broth cultures were centrifuged and the pellets resuspended in water. The parental mycelial suspensions were mixed in an approximate 1:1 ratio based on optical density and spread $(0.2 \mathrm{ml})$ on a large slant of complete agar $(\mathrm{CM})(15 \mathrm{ml})$.

After 6 days of incubation the surface of the mixed culture was scraped with a loop into $10 \mathrm{ml}$ of water. The resulting suspension was shaken vigorously with quartz pebbles, washed by centrifugation and resuspended in water. This suspension was then plated $(0.1 \mathrm{ml})$ at $10^{-1}, 10^{-2}$ and $10^{-3}$ dilutions on supplemented MM plates, each medium being selective for one allele from each parent, and at $10^{-5}$ and $10^{-8}$ dilutions on appropriately supplemented MM plates to determine the titer of the parent strains in the culture of the cross.

Test for New Rifamycins Produced by Recombinant Strains

A loopful of cell mass from parental or recombinant strains grown on complete agar was inoculated into $20 \mathrm{ml}$ of medium 148 in $50 \mathrm{ml}$ Erlenmeyer flasks. The cultures were incubated at $28^{\circ} \mathrm{C}$ on a rotary 
shaker for 7 days at $250 \mathrm{rev}$./minute. The culture broth was then filtered, adjusted to $\mathrm{pH} 2$ with $\mathrm{HCl}$ and extracted once with an equal volume of methylene chloride. The organic phase was concentrated 20 -fold and analysed by thin-layer chromatography (Merck silica gel $60 \mathrm{~F}_{254}$ plates). After developing with chloroform - methanol, $4: 1$, new products could be distinguished from the rifamycins produced by the parental strains and rifamycin standard substances (rifamycins $\mathrm{B}, \mathrm{O}, \mathrm{S}, \mathrm{W}$, protorifamycin I) which were run together on the same chromatogram.

Fermentation

The fermentation of $N$. mediterranei R-21 was carried out on a 300-liter scale with liquid complex medium $151 \mathrm{~b}$ with the addition to the medium before sterilization of SAG $4710.375 \mathrm{~g} / \mathrm{liter}$ and UCON LB $6250.125 \mathrm{~g} /$ liter as antifoam agents. The inoculum cycle was as follows: $0.1 \% \mathrm{v} / \mathrm{v}$ of laboratory pre-culture grown in liquid complex medium 148 was inoculated into a fermentor containing 30 liters of medium $148 \mathrm{c}$ as the seed culture step, duration 48 hours, $28^{\circ} \mathrm{C}$, aeration $11 /$ liter/minute, pressure 0.5 bar, agitation $600 \mathrm{rpm}$, single 6-bladed turbine agitator, 4 baffles; $5 \% \mathrm{v} / \mathrm{v}$ of the seed culture was inoculated into 300 liters of medium $151 \mathrm{~b}, 28^{\circ} \mathrm{C}, 450 \mathrm{rpm}(1 \sim 2$ watts/liter, single six-bladed turbine agitator), 4 baffles, aeration 1 1/liter/minute, pressure 0.5 bar, 168 hours duration.

\section{Transformation Assay}

Shake flasks $(50 \mathrm{ml})$ with $20 \mathrm{ml}$ of medium 148 were inoculated with a loopful mycelium of strain $\mathrm{R}-21$. The culture was incubated on a rotary shaker at $250 \mathrm{rev} . /$ minute for 4 days. The mycelium was then washed twice with fresh medium 148 and resuspended in shake flasks with $20 \mathrm{ml}$ medium 148 . For the transformation assay rifamycin $\mathrm{S}$ was added to give a final concentration of $100 \mu \mathrm{g} / \mathrm{ml}$ and the culture was incubated for 24 or 48 hours. Control cultures (de novo synthesis) were made in which no rifamycin $\mathrm{S}$ was added. The culture broth was extracted with methylene chloride and analysed by thin-layer chromatography as already described.

\section{Experiments and Results}

\section{Isolation of the Recombinant Strain R-21}

Strain R-21 was isolated as a recombinant colony derived from an intraspecific cross between the two Nocardia mediterranei strains T104 and T191. The origin of the two parental strains is shown in Fig. 2. The parental strain T104 is itself a recombinant strain, which was selected for its properties as a multimarked strain with adequate rifamycin B production. The parental strains of T104 were N813, an industrial high rifamycin B producer strain and the multimarked strain T96, a very low rifamycin B producer. The rifamycin W producing parental strain T191 is a UV-light induced mutant (ans-11) of the high rifamycin B producer FS 713. It is blocked in the transformation of rifamycin $\mathrm{W}$ to $\mathrm{S}$ and produces rifamycin $\mathrm{W}$ and no rifamycin $\mathrm{B}$.

After having crossed the strains T104 and T191 on a slant of CM agar the resulting suspension of mycelium was plated on MM media selecting separately for one of the three alleles of T191 cys $-3^{+}$, lys- $1^{+}$, leu- $1^{+}$and the str-2 allele of T104. A random sample from the recombinant colonies
Fig. 2. Family tree of strain Nocardia mediterranei R-21.

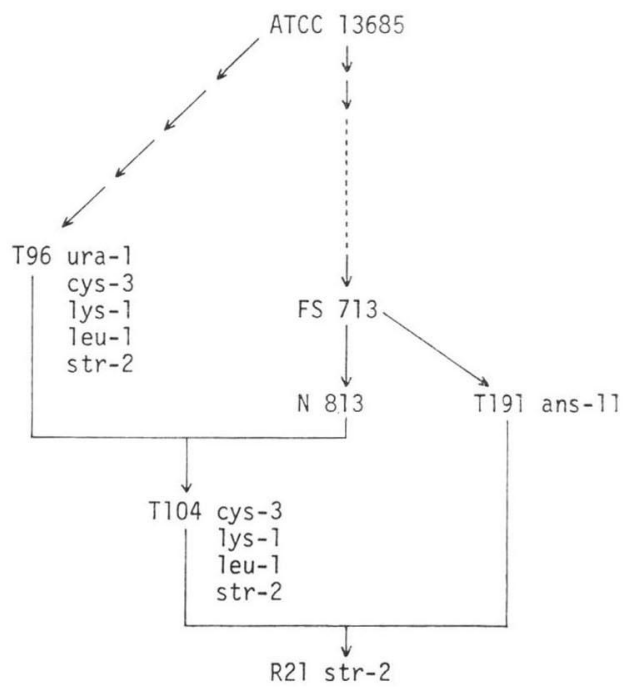


Table 1. Cross T104 cys-3- lys-1- leu-1- str-2 $\times$ T191 ans-11 (Rifamycin W).

\begin{tabular}{|c|c|c|c|c|c|}
\hline \multirow{2}{*}{$\begin{array}{l}\text { Selected } \\
\text { alleles }\end{array}$} & \multirow{2}{*}{$\begin{array}{l}\text { Recombinants } \\
\text { per ml suspension }\end{array}$} & \multirow{2}{*}{$\begin{array}{l}\text { Recombinant } \\
\text { frequency }{ }^{1)}\end{array}$} & \multicolumn{3}{|c|}{ Isolated recombinants producing: } \\
\hline & & & Rifamycin B & Rifamycin W & $\begin{array}{l}\text { New products } \\
\text { visible on TLC }\end{array}$ \\
\hline leu- $1^{+} /$str-2 & $2.4 \times 10^{5}$ & $1.2 \times 10^{-3}$ & 108 & 3 & $1^{2)}$ \\
\hline lys $-1^{+} /$str-2 & $1.9 \times 10^{5}$ & $0.9 \times 10^{-3}$ & 76 & 0 & 0 \\
\hline cys $-3^{+} /$str- 2 & $2.4 \times 10^{5}$ & $1.2 \times 10^{-3}$ & 106 & 0 & $1^{3)}$ \\
\hline $\begin{array}{l}\text { 1) Calc } \\
\text { per } \\
\text { 2) Recc } \\
\text { 3) Rec }\end{array}$ & $\begin{array}{l}\text { as recombinants } \\
\mathrm{T} 191 \text { and } 1 \times 10^{9} \mathrm{p} \\
\text { ant strain } \mathrm{R}-21 \text {. } \\
\text { lant strain } \mathrm{R} 69 \text {, see }\end{array}$ & $\begin{array}{l}\text { minority paren } \\
1 \text { for T104. } \\
\text { ussion. }\end{array}$ & The titers & the parental & rains were $2 \times 10$ \\
\hline
\end{tabular}

grown after 10 days of incubation on these selective plates was picked off with sterile toothpicks and streaked on the master plates ( 30 per plate) containing the same selective medium. After 8 days of incubation, the master plates were replicated onto plates of CM agar. These CM plates were incubated for 7 days and then a loopful of cell mass from each well grown patch was grown up in liquid medium 148 in order to test for rifamycin production. The results of the cross are shown in Table 1.

Characterization of Recombinant Strain R-21 and its Fermentation Products

The growth requirements of the recombinant strain R-21 were determined by plating on suitably supplemented MM agar. The strain was found to be prototrophic and streptomycin resistant. This showed that it had received the str- 2 allele from the parent strain T104 and the three alleles cys- $3^{+}$, lys $-1^{+}$and leu- $1^{+}$from the parent strain T191. The morphology of strain R-21 when grown on agar or in liquid medium is the same as that of the two parental strains. It does not sporulate under our conditions and the mycelium tends to break up into rod like fragments of $5 \sim 20 \mu$ length. For a first characterization of the fermentation products, strain R-21 was fermented in shake flasks with medium 148 and the culture broth was extracted as described in Materials and Methods. Thin-layer chromatogram analysis showed that recombinant strain R-21 produces a mixture of new products in medium 148 as well as rifamycin $\mathrm{B}$ and small amounts of the rifamycins $\mathrm{O}, \mathrm{S}, \mathrm{W}$ and $\mathrm{P}$. The identity of these known rifamycins could be proved by purification and thin-layer chromatography using different solvent systems for development. In order to test for antibiotic activity the thin-layer chromatogram was replicated on agar plates containing a top layer of the test organisms Sarcina lutea ATCC 9341 or Escherichia coli 205. After incubation for 24 hours at $37^{\circ} \mathrm{C}$ the resultant inhibition zones were analysed. The mixture of new rifamycins was active against Sarcina lutea ATCC 9341 and one substance (3-hydroxyrifamycin S) was also active against Escherichia coli 205.

Fermentation and Isolation

To obtain larger amounts of the new products of strain R-21 a fermentation was carried out on a 300 -liter scale with liquid complex medium 151b. After 168-hour fermentation the culture broth was filtered through celite and the filtrate was extracted five times with chloroform at $\mathrm{pH} 6.5$. The combined chloroform extracts were washed with water, dried and evaporated to dryness leaving $396 \mathrm{~g}$ of reddish brown residue of rifamycin mixture. The remaining aqueous solution was then acidified to pH 2.5 and extracted five times with ethyl acetate. The combined ethyl acetate extracts were washed with water, dried and evaporated to dryness leaving $413 \mathrm{~g}$ of a yellow residue containing mainly rifamycin B. 
Table 2. Rf values of the rifamycins of recombinant strain R-21 on thin-layer chromatography.

\begin{tabular}{l|c|c|c|c}
\hline \multirow{2}{*}{ Compound } & \multicolumn{4}{|c}{ Solvent system } \\
\cline { 2 - 5 } & A & B & C & D \\
\hline 3-Hydroxyrifamycin S & 0 & 0.09 & 0.19 & 0.27 \\
3,31-Dihydroxyrifamycin S & 0 & 0.05 & 0.09 & 0.19 \\
16,17-Dehydrorifamycin G & 0.02 & 0.17 & 0.33 & 0.29 \\
Rifamycin W-lactone & 0.07 & 0.48 & 0.26 & 0.35 \\
Rifamycin W-hemiacetal & 0.14 & 0.49 & 0.39 & 0.34 \\
30-Hydroxyrifamycin W & 0.09 & 0.48 & 0.33 & 0.29 \\
28-Dehydroxymethyl-28,30- & 0.03 & 0.31 & 0.31 & 0.39 \\
dihydroxyrifamycin W & 0.33 & 0.61 & 0.69 & 0.27 \\
\hline Rifamycin S & 0 & 0.15 & 0.22 & 0.31 \\
Rifamycin G & 0 & 0.13 & 0.09 & \\
Rifamycin W & & & & \\
\hline
\end{tabular}

Solvent system: A Ethyl acetate - cyclohexane (2:1).

B Ethyl acetate - acetone - water $(72: 24: 4)$.

C Chloroform - methanol (9:1).

D Toluene - acetone - methanol $(5: 3: 2)$.

Further separation into its components of the crude rifamycin mixture of the pH 6.5 extraction was achieved by chromatography on silica gel $(12 \mathrm{~kg})$ with chloroform and increasing amounts of methanol as eluants. Thirty 10-liter fractions were collected. 3-Hydroxyrifamycin $\mathrm{S}$ was enriched in fractions 12 and 13, 3,31-dihydroxyrifamycin $\mathrm{S}$ in fractions 19 22; 16,17-dehydrorifamycin $\mathrm{G}$ in fractions $16 \sim 18$ and the ansamycins of the rifamycin $W$ type in fractions $14 \sim 18$.

Final purification of the enriched rifamycins was achieved either by repeated chromatography on silica gel or, in some cases, Sephadex LH-20 columns (with methanol as eluant) or by preparative TLC on silica gel $\mathrm{PF}_{254}$ plates (Merck, $1.5 \mathrm{~mm}$ layer) (Table 2).

\section{Transformation Studies}

In order to test if 3-hydroxyrifamycin $\mathrm{S}$ is a metabolic derivative of rifamycin $\mathrm{S}$, transformation studies were performed. Washed mycelium of strain R-21 was used and the transformation medium was 148 . The analysis of the thin-layer chromatograms of the extracted cultures showed that strain R-21 transformed $50 \%$ of the added rifamycin S to rifamycin B in 24 hours and $90 \%$ in 48 hours. No transformation to 3-hydroxyrifamycin $\mathrm{S}$ is detectable under these conditions. The small amount of 3-hydroxyrifamycin $\mathrm{S}$ found in the cultures is the result of de novo synthesis and not transformation, since it is detectable in equal amounts in the transformation mixture (rifamycin $\mathbf{S}$ added) and the control (no rifamycin $\mathrm{S}$ added).

\section{Discussion}

We have shown that it is possible to obtain a strain that produces new rifamycins by means of intraspecific recombination of two $N$. mediterranei mutants. The new rifamycins found were not detected in the fermentation broth of either of the parental strains when the same analytical methods were applied. Since we are dealing with an intraspecific recombination system using mutant strains derived from one ancestor strain ( $N$. mediterranei ATCC 13685) the genotype of the parental strains differed only in the mutations introduced in the laboratory (Fig. 2). 
We therefore believe that the new rifamycins found are the result of a different pattern of enzyme activities brought about by genetic recombination. The enzymes responsible for the synthesis of the new rifamycins are very probably also present in the parental strains but their activity is so low that their products are not detectable with our analytical methods. One possible reason for the considerable range of new products found is that three strains of the direct rifamycin strain development line are involved in the recombination system used, namely, the wild type strain ATCC 13685 and two highly developed production mutants FS713 and N813. Therefore many mutations introduced during the strain development of $N$. mediterranei may have been substituted by the wild type allele through the recombination event, thus leading to a great variability of genes involved in rifamycin biosynthesis.

In addition to strain R-21 another recombinant strain (R69) was found which also produced a number of different substances visible on the thin-layer chromatogram (Table 1). When a comparison of these substances was made with those produced by recombinant strain R-21 using thin-layer chromatography with different solvent systems for development, it could be shown that the mixture of rifamycins was the same in both strains. Strain R69 was not analysed further.

The transformation experiments were performed in order to study the biogenetic origin of 3hydroxyrifamycin $\mathrm{S}$ and to find out if it could be produced by biotransformation from rifamycin $\mathrm{S}$. The results obtained indicated that 3-hydroxyrifamycin $\mathrm{S}$ is not a metabolic derivative of rifamycin $\mathrm{S}$ which is formed by hydroxylation at carbon atom 3. The hydroxyl group must therefore be introduced at an earlier stage in rifamycin biosynthesis.

\section{Acknowledgement}

The authors thank Mrs. I. OBERKIRCH for her technical assistance.

\section{References}

1) Lancini, G. \& C. Hengeller: Isolation of rifamycin SV from a mutant Streptomyces mediterranei strain. J. Antibiotics 22: 637 638, 1969

2) Martinelli, E.; G. G. Gallo, P. Antonini \& R. J. White: Structure of rifamycin W, a novel ansamycin from a mutant of Nocardia mediterranei. Tetrahedron 30: 3087 3091, 1974

3) Ghisalba, O.; P. Traxler \& J. NüEsch: Early intermediates in the biosynthesis of ansamycins. I, Isolation and identification of protorifamycin I. J. Antibiotics 31:1124 1131, 1978

4) Lancini, G.; C. Hengeller \& P. Sensi: New naturally occurring rifamycins. Progr. Antimicr. Anticancer Chemoth., Proc. Int. Congr. Chemoth., Vol. 2, pp. 1166 1173, Univ. Park Press, Baltimore, Md., 1970

5) White, R. J.; G. Lancini \& P. Sensi: Proceedings of the First Intersectional Congress of IAMS. Ed. T. Hasegawa, p. 483, Science Council of Japan, 1975

6) Ghisalba, O.; P. Traxler, H. Fuhrer \& W. J. Richter: Early intermediates in the biosynthesis of ansamycins. II. Isolation and identification of proansamycin B-M1 and protorifamycin I-M1. J. Antibiotics 32: 1267 1272, 1979

7) Ghisalba, O.; P. Traxler, H. Fuhrer \& W. J. Richter: Early intermediates in the biosynthesis of ansamycins. III. Isolation and identification of further 8-deoxyansamycins of the rifamycin-type. J. Antibiotics 33: 847 856, 1980

8) Schupp, T.; R. Hütter \& D. A. Hopwood: Genetic recombination in Nocardia mediterranei. J. Bacteriol. $121: 128 \sim 136,1975$

9) Schupp, T.; P. Traxler \& J. NÜesch: Europ. Pat. Appln. 14,181, 1980

10) Traxler, P.; T. Schupp, H. Fuhrer \& W. J. Richter: 3-Hydroxyrifamycin S and further novel ansamycins from a recombinant strain R-21 of Nocardia mediterranei. J. Antibiotics 34: 971 979, 1981 